\title{
ANALYSIS OF INERTIAL SENSORS FOR THE REQUIREMENT TO CONTROL A SMALL UNMANNED AIRPLANE
}

\author{
Dávid MEGYESI*, Róbert BRÉDA, Michal SCHREINER \\ Technical University of Košice, Faculty of Aeronautics, Rampová 7, 04121 \\ *Corresponding author. E-mail: david.megyesi@tuke.sk
}

\begin{abstract}
The article deals with the analysis of angular velocity sensors, for the control of a small unmanned airplane. In the paper, the authors discuss the process of creating an angular velocity sensor error model for the analysis of the interfering component size of the useful signal. The built-in error model of the inertial sensor will be implemented in a small, unmanned airplane model, in order to increase the stability and controllability of the airplane. Allan variation will be used to analyze stochastic processes of inertial sensors.
\end{abstract}

Keywords: Sensor, noise, Allan variance

\section{INTRODUCTION}

The article deals with the issue of sensor error model design, with which it will be possible to simulate errors in any IMU unit that can be installed in a small unmanned aircraft. With this model, Kallman filters can be designed to help optimize airplane stability and controllability criteria. Allan variation is used to analyze the stochastic processes of sensors. The prerequisite for this is the possibility of designing filters for a range of IMU units that can be installed in a small unmanned aircraft to increase the stability and controllability of the airplane using different IMUs.

\section{SMALL UNMANNED AIRPLANE AS A OBJECT OF CONTROL}

The aircraft may be presented as an object defined by a series of coordinates relating to its axes. This object has six degrees of freedom, three of which are translational and three are rotational. Translational degrees of freedom define the movement along the aircraft axis and the rotational motion around the aircraft axis.

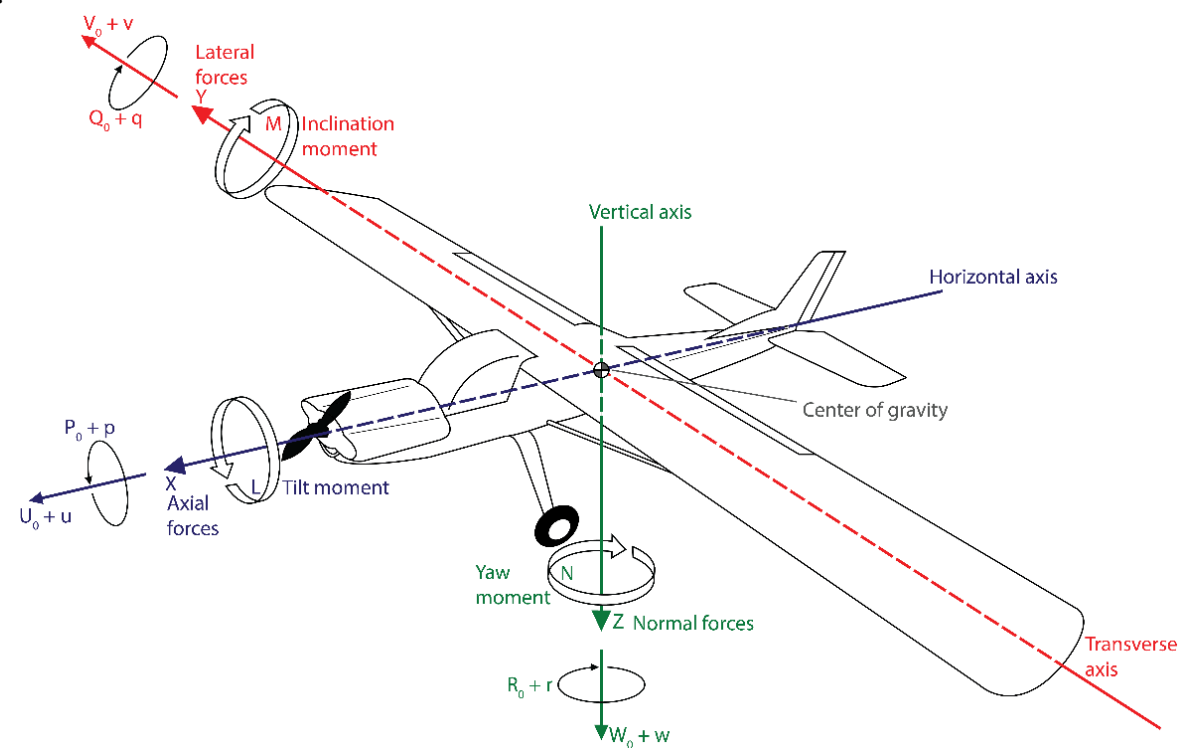

Figure 1 Representation of the coordinates of a small unmanned aircraft 
Figure 1 shows a model of a small unmanned aerial vehicle "SkyDog", showing the coordinates related to the airplane axes. $\mathbf{U}_{\mathbf{0}}, \mathbf{V}_{\mathbf{0}}, \mathbf{W}_{\mathbf{0}}, \mathbf{P}_{\mathbf{0}}, \mathbf{Q}_{\mathbf{0}}$ and $\mathbf{R}_{\mathbf{0}}$ are translational and rotational speeds in steady state aircraft; $\mathbf{u}, \mathbf{v}, \mathbf{w}, \mathbf{p}, \mathbf{q}$ and $\mathbf{r}$ are small disturbing changes of these speeds.

In this case, we are talking about a non-linear model aircraft that includes another model. These models include a model of aircraft performance members (actuators), a model of aircraft aerodynamics, a model of aircraft propulsion (engine), an atmosphere model, and an aircraft sensor model.

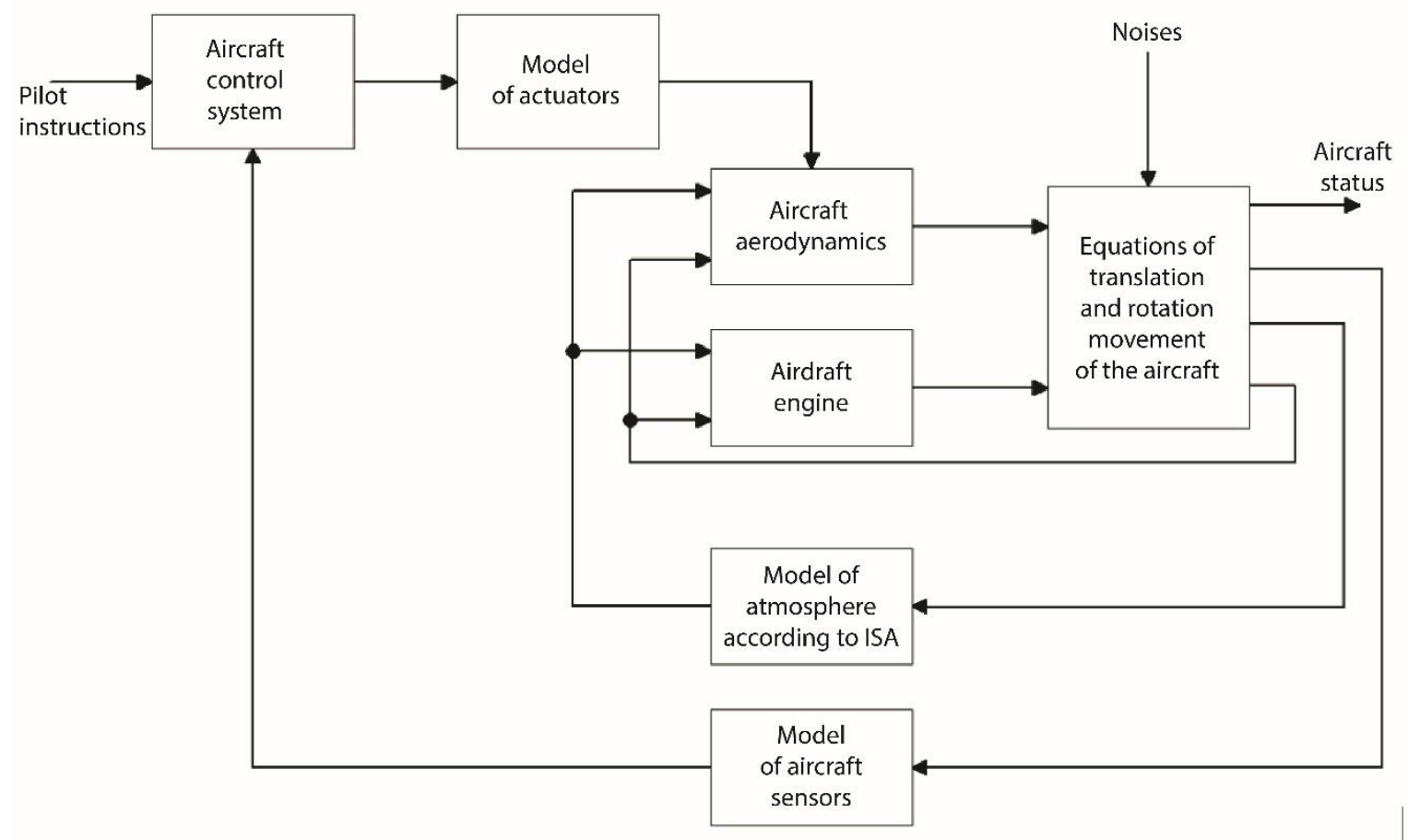

Figure 2 Nonlinear aircraft model

It is therefore possible to say that the stability of the aircraft is also influenced by the atmosphere surrounding it during the flight, the aerodynamics of the aircraft engine of the aircraft, the actuators acting on the aircraft control surfaces and also the sensors used in the control system feedback. Sensors whose data enter the aircraft control system provide feedback on the value of the desired angular velocity, acceleration, or rate. Based on this information, the control system knows the correctness of the control operation performed and, if necessary, adjust the control signal value so that the angular velocity in the individual axes at stable values is the end result.

The rotating and sliding movement of the aircraft is based on its dynamics and equations of motion. Motion equations of the plane are based on Newton's second law of motion. The dynamics of movement of the aircraft around the center of gravity is based on the basic equation of moments valid for a rigid body. Derived equations for rotary and sliding motion are also implemented in the 6DOF ECEF block, which is part of the Matlab Simulink "AerospaceBlockset" library.

\section{AIRCRAFT SENSOR MODEL}

The creation of a sensor mathematical model is needed for the design of automatic control algorithms. In this control, information from the sensors is included in the feedback in which, in addition to the required information, there are also various kinds of noise and interference. Since these noises and interference are also handled by management systems, their identification is necessary. The sensor error model is used for the purpose of sensor error identification. When creating a sensor error model, a long-term static measurement is required to identify sensor errors. The basic angular velocity sensor 
model is defined as the output of the sensor $\boldsymbol{g}_{\boldsymbol{r}}(\boldsymbol{t})$, which is equal to the angular velocity of the object $\boldsymbol{r}$, bias $\boldsymbol{b}$, bias drift $\boldsymbol{b}_{\boldsymbol{r}}(\boldsymbol{t})$ and the wideband sensor noise $\boldsymbol{w}_{\boldsymbol{g}}$.

$$
g_{r}(t)=r+b+b_{r}(t)+w_{g}
$$

The sensor output, with which we create the error model, contains other noise mechanisms. However, the factors listed in equation (1) are considered significant in this category of sensors. If we suppress the bias in the sensor by introducing the obtained calibration value, we will remove this unidirectional component and, with respect to equation (1), convert it to form:

$$
g_{r}(t)=b_{r}(t)+w_{g}
$$

For reconstruction according to standards [1] and [2], standard deviation of wideband noise, bias drift-time correlation, as well as its standard deviation should be determined. Bias drift is modeled as a first order Gauss-Mark process. With the help of the Allan variation graph and the relevant relationships it is possible to determine the necessary parameters. The basic parameter to be determined is the standard deviation of wideband noise, also known as "Angular Random Walk" (ARW). On a logarithmic graph of Allan's variation, this noise mechanism has a $-\frac{1}{2}$ slope defined by the standard. Its value can be determined by the relationship:

$$
\sigma_{g}=\frac{N}{\sqrt{\Delta t}}
$$

In this relation, $\boldsymbol{N}$ is defined as a wideband noise coefficient and $\boldsymbol{\Delta t}$ determines the sampling time, which in our case is $0,01 \mathrm{~s}$.

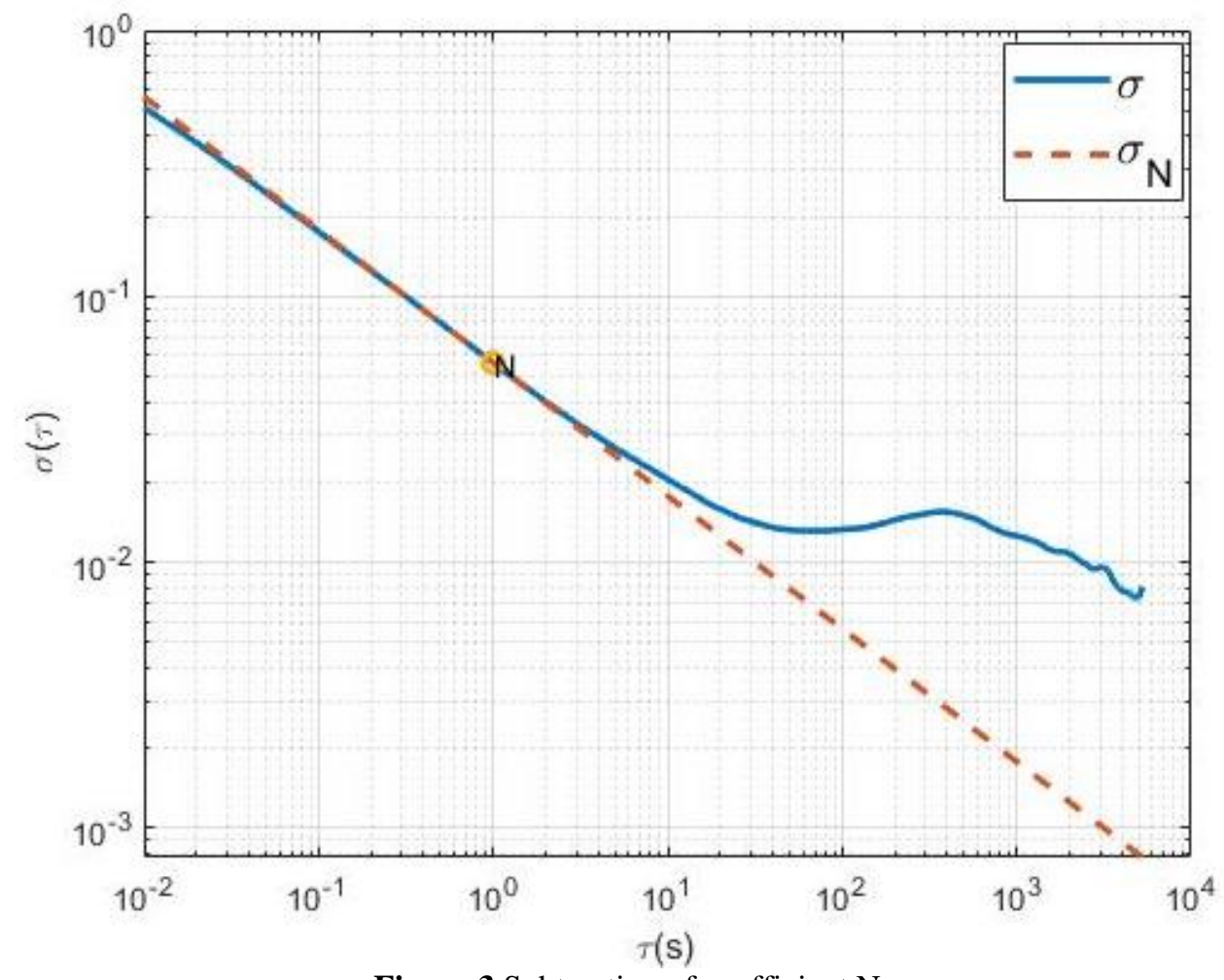

Figure 3 Subtraction of coefficient $\mathrm{N}$ 
Using equation (3), we get the value of the standard deviation of wideband noise at:

$$
\sigma_{g}=0,562 \% \sec
$$

For comparison, after applying std () in the MATLAB environment, the sensor output is:

$$
\sigma_{g}=0,5297^{\circ} / \mathrm{sec}
$$

For the determination of bias drift is determined by the following formulation, according to which the standard deviation of uncorrelated wideband noise is significantly greater than correlated noise. Separation of noise mechanisms is the easiest to do by dividing signal mixtures by lower filtration. Using the recommendations and procedures in [3], the bottom file filtering was converted from a filter window to 100 samples. The result of the filtration is shown in figure 4 . Figure 5 shows the allan graphs of the filtered and unfiltered signal that are used for comparison.

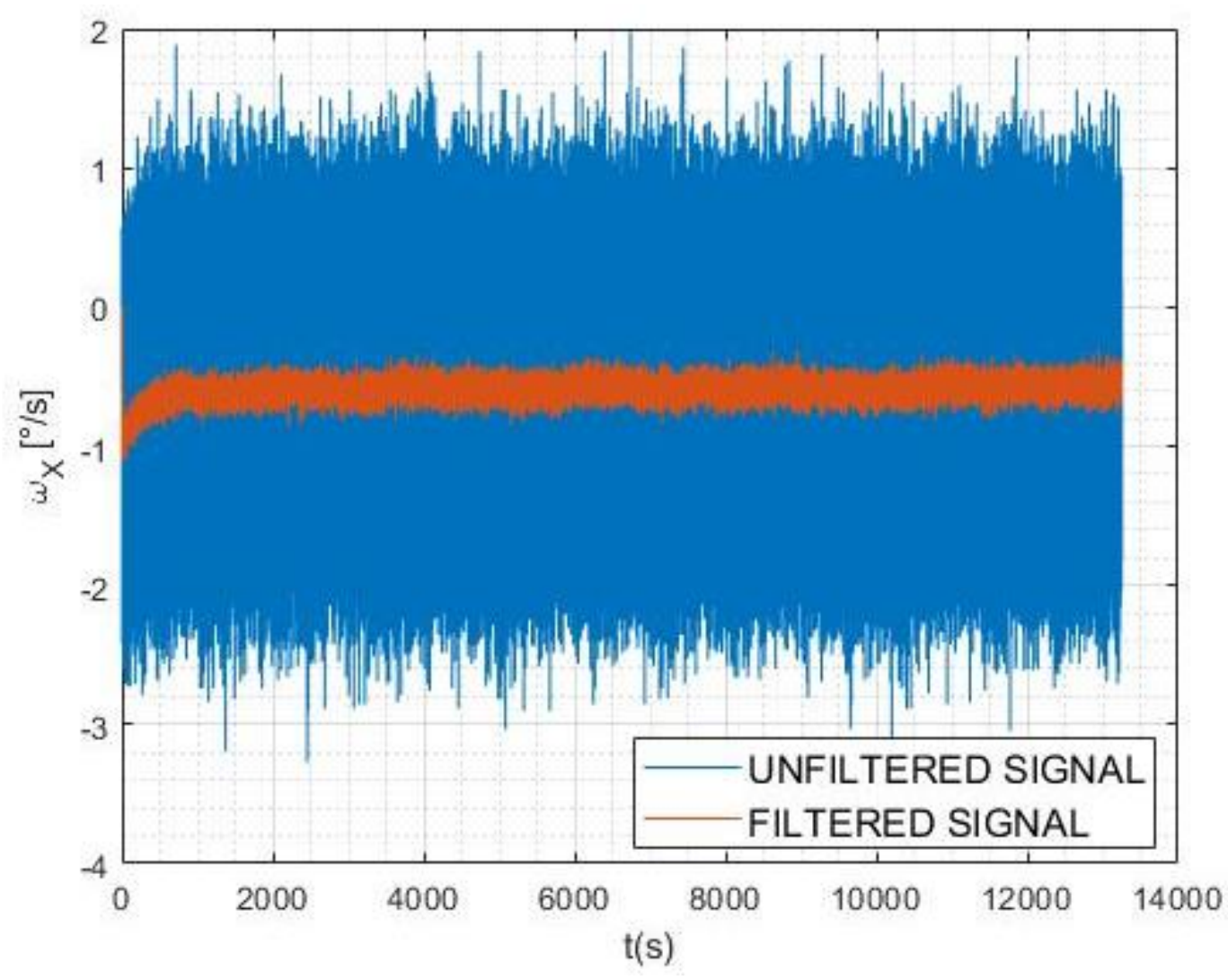

Figure 4 Filtration result according to procedures in [50] 


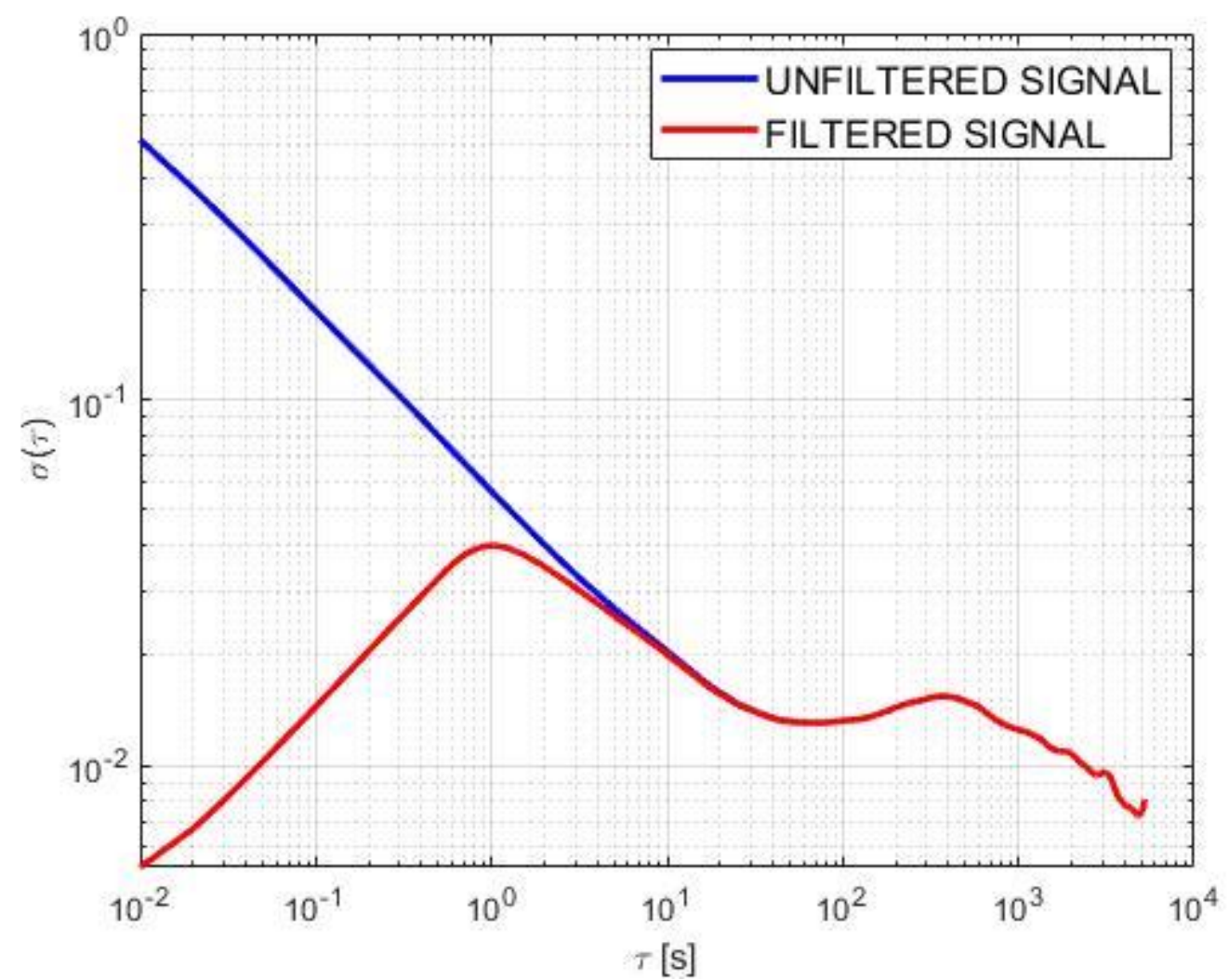

Figure 5 Allan variation of original and filtered signal

It is possible to see in the figure the separation of the part copying the region of instability bias and the subsequent maximum of the curve determining the correlation time. The determination of the correlation time is defined by:

$$
\tau=1,89 T_{C}
$$

The maximum determined from the graph of Allan variation in has value:

$$
\tau=366,53 \mathrm{sec} .
$$

As a result, the correlation time value can be determined as follows:

$$
T_{C}=\frac{\tau}{1,89}=193,9312 \mathrm{sec}
$$

A coefficient B is used to determine Bias instability. For this coefficient:

$$
B=\frac{\min }{0,664}
$$




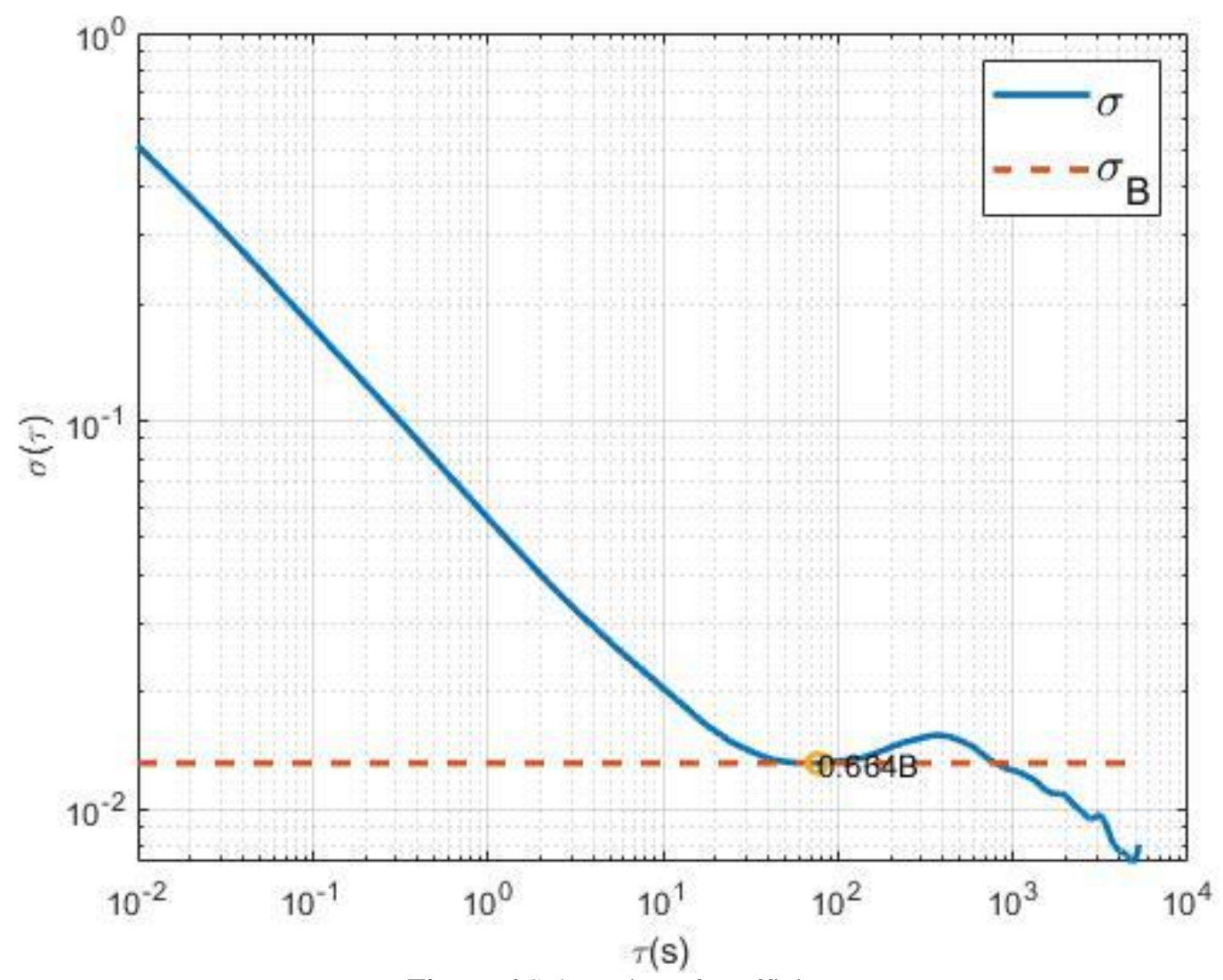

Figure 6 Subtraction of coefficient B

The Bias instability value is by (9) then:

$$
B=\frac{0,013114}{0,664}=0,01975 \% \text { sec }
$$

As mentioned above, the bias drift is modeled as a first order Gauss-Markov process whose behavior can be described:

$$
x_{n+1}=e^{-\beta \Delta t} \cdot x_{n}+\sqrt{\sigma_{G M}^{2}\left(1-e^{-2 \beta \Delta t}\right)} \cdot w_{n}
$$

In the calculation there is a previous signal sample $\boldsymbol{x}_{\boldsymbol{n}}$, white noise $w_{n}$ and constants $\boldsymbol{\beta}, \boldsymbol{\Delta t}$ and $\boldsymbol{\sigma}_{\boldsymbol{G M}}$. Constant $\Delta \boldsymbol{t}$ represents the step between the samples and its value is the same sampling rate, which in our case is $0.01 \mathrm{~s}$. The constant $\boldsymbol{\sigma}_{G M}$ is calculated as the standard deviation of the filtered signal shown in figure 4. The constant $\boldsymbol{\beta}$ is calculated using the relation (12), which includes the already known correlation time value $\boldsymbol{T}_{\boldsymbol{C}}$.

$$
\beta=\frac{1}{T_{C}}
$$


Based on the relation (11) we can build a simulation simulation in the MATLAB Simulink that simulates the first order Gauss-Mark process. We generate white noise in the simulation circuit using a white noise generator block. The Gauss-Mark model is shown in the following figure.

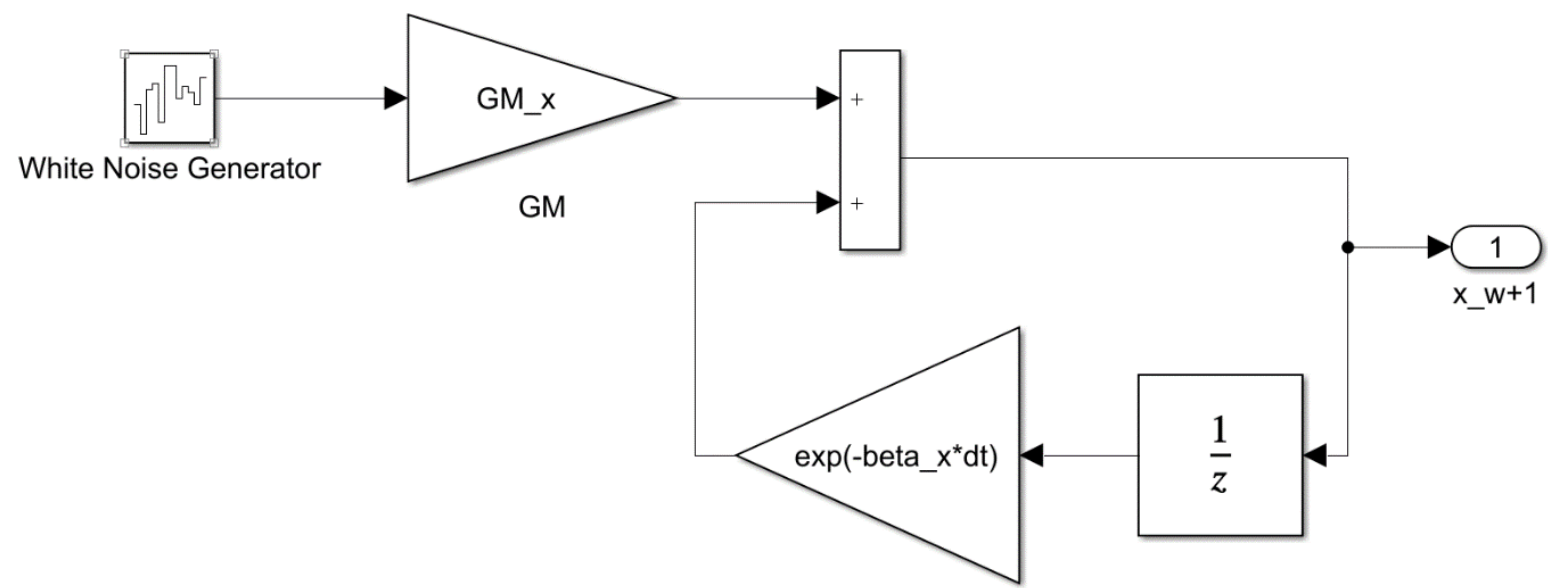

Figure 7 Model of Gauss-Mark process of first order simulating Bias Drift

The last component is a wideband sensor noise or an angle random walk (ARW). In the sensor model, we create a white noise generator block. The square of the value given in the graph is inserted into the field determining the power of this generator. The resulting sensor model following these steps is shown in figure 8.

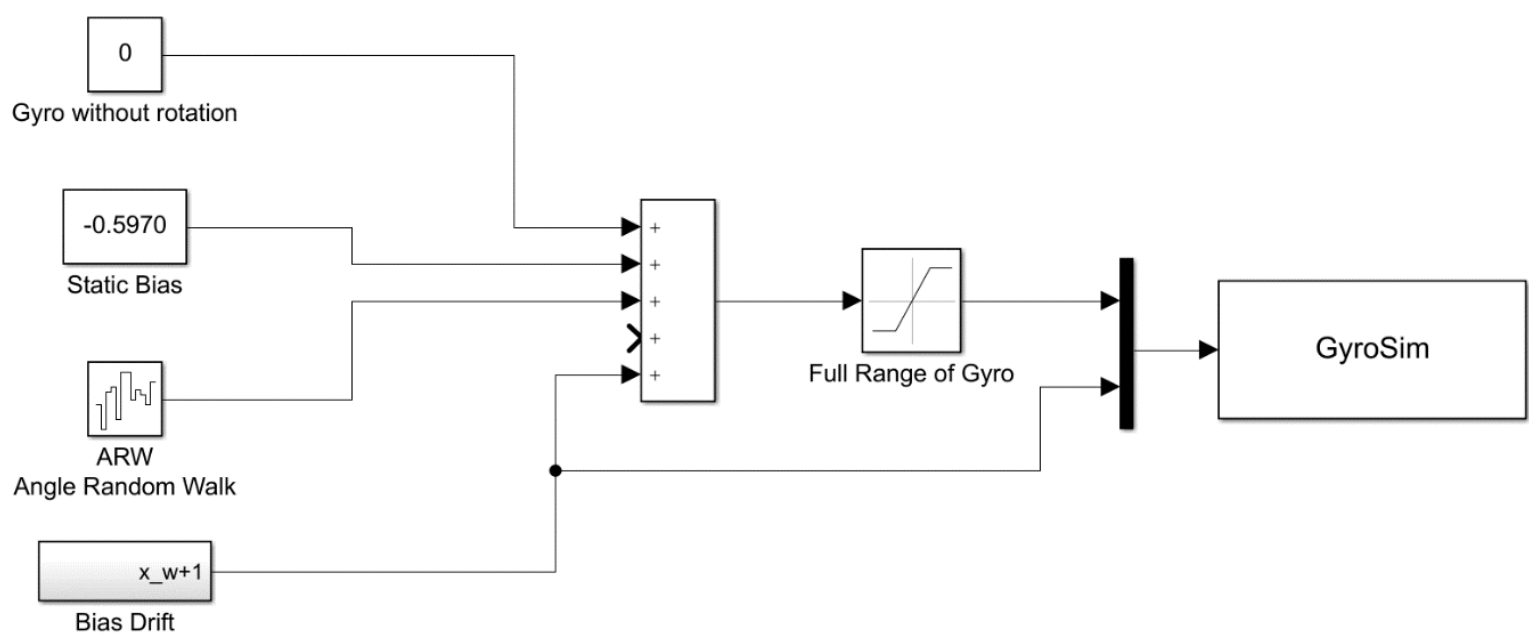

Figure 8 Simple model of angular velocity sensor

The result is a comparison of real data measured during static measurement with data simulated with the created model. The similarity of the real data with the simulated data is shown by the comparison of the bias values of the two signals. In the case of real data, the bias value is $-0,5970 \% \mathrm{~s}$ and in the case of the simulated signal the bias value is $-0,5909 \%$. 


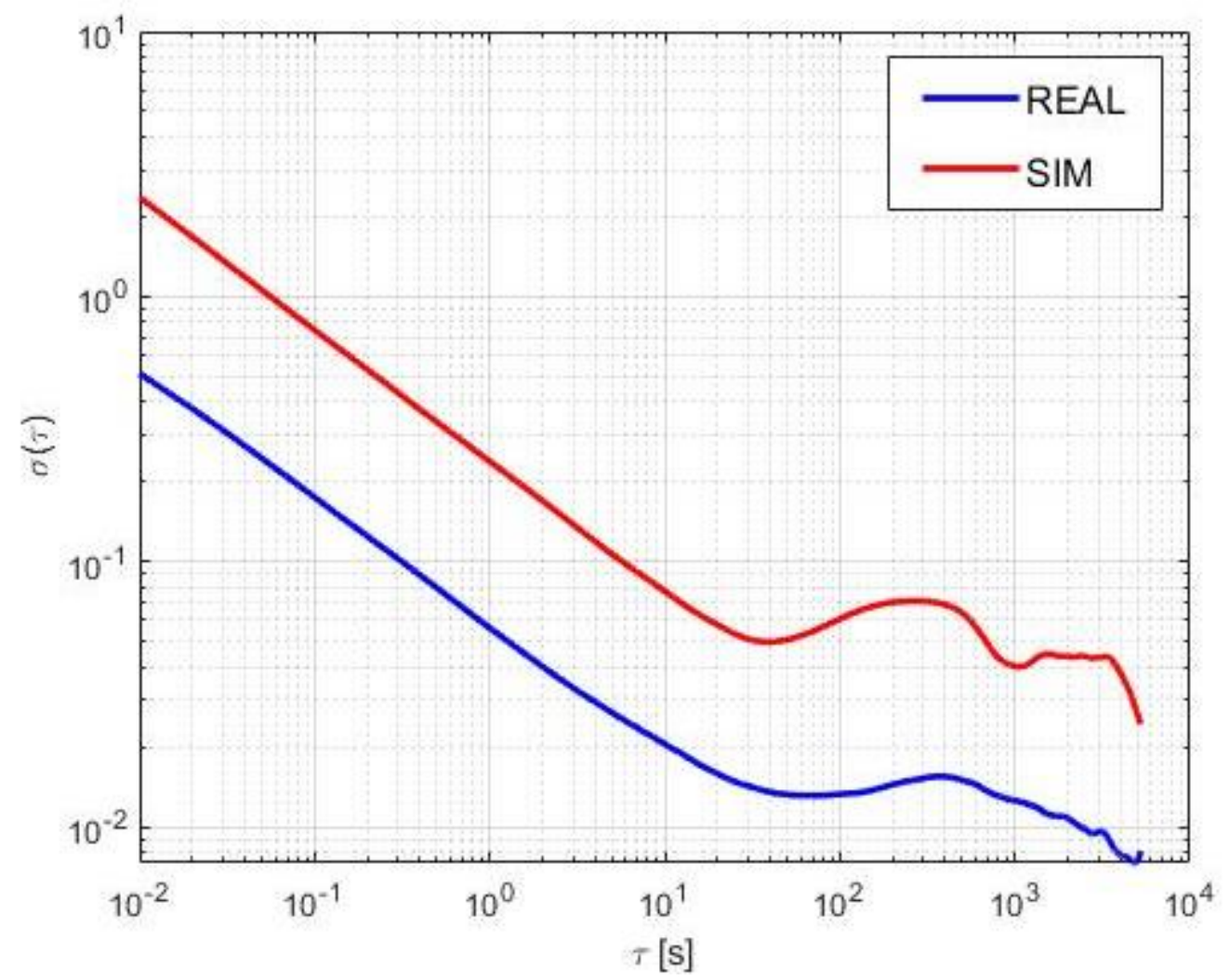

Figure 9 Allan's variations of real and simulated data

The standard deviation of the wideband sensor noise is increased in terms of the information in [1]. This ensures coverage of possible unstable measurement conditions that reduce the quality of modeling outputs. These conditions include temperature change, vibration, other noise mechanisms, and the like.

\section{CONCLUSION}

The goal of the work as a whole is to design and simulate the architecture that filters out interfering signals from the control process, introducing sensors as well as unpredictable phenomena during flight, such as sudden gusts of wind. To verify the overall architecture, it is necessary to design a comprehensive sensor model to simulate all measurements interfering with measurements. The goal is to propose, in addition to a comprehensive error model, a way to filter out these noises, provided that the stability and controllability of the airplane during flight is not reduced. As described in the previous chapter for the noise analysis of sensors of the inertial measuring unit, it will be necessary to use tools for stochastic and deterministic parameters of sensors and also to design the error model of sensors with their subsequent simulation. Based on the proposed error model, it will be possible to design Kalman filters for a small unmanned aircraft control system and incorporate them into the circuit to optimize the stability and controllability criteria of the airplane.

\section{References}

[1] Flenniken, Warren S. Modeling Inertial Measurement Units and Analyzing the Effect of their Errors in Navigation Applications. Master of Science Thesis, Auburn University, Alabama 2005.

[2] IEEE Standard Specification Format Guide and Test Procedure for Single-Axis Interferometric Fiber Optic Gyros, IEEE Std 952- 1997(R2003) Annex. B (2003).

[3] Demoz, Gebre - Egziabher. Design and Performance Analysis of a Low - Cost Aided Dead Reckoning Navigator. Dissertation thesis. Department of Aeronautics and Astronautics of Stanford University. 2004. 
[4] Freescale Semiconductor, Inc., Allan variance: Noise analysis for gyroscopes, 2015.

[5] SOTÁK M., BRÉDA R.: Removing the high frequency noise components from gyro and accelerometer measurement, Acta Avionica, Roč. 10, č. 5, 2008, s. 36-40. - ISSN 1335-9479

Received 12, 2019, accepted 07, 2020

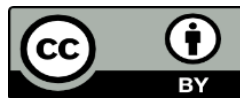

Article is licensed under a Creative Commons Attribution 4.0 International License 